\title{
Supporting Information: Semiconducting Bipolar Membranes: Photochemical Salt Pumps
}

\author{
Fuding Lin and Mark C. Lonergan*
}

Department of Chemistry and Biochemistry, University of Oregon, Eugene, OR 97403.

\section{Experimental}

\section{1 $\quad \mathrm{PA}_{\mathrm{A}} / \mathrm{PA}_{\mathrm{C}} \mathrm{SBM}$ fabrication}

The $\mathrm{PA}_{\mathrm{A}}$ and $\mathrm{PA}_{\mathrm{C}}$ were synthesized as described previously ${ }^{1}$ using ring-opening metathesis polymerization of ionically functionalized cyclooctatetraenes. The polymerizations result in the tetramethylammonium salt of $\mathrm{PA}_{\mathrm{A}}$ and the tetrafluoroborate salt of $\mathrm{PA}_{\mathrm{C}}$, and samples were available from a prior study. The $\mathrm{PA}_{\mathrm{A}} / \mathrm{PA}_{\mathrm{C}}$ bilayer was fabricated on a 1 in $\mathrm{x} 2$ in glass microscope slide by sequential spin-coating of a $10 \mathrm{mg} / \mathrm{mL}$ solution of $\mathrm{PA}_{\mathrm{C}}$ in dimethylformamide followed by a 10 $\mathrm{mg} / \mathrm{mL}$ solution of $\mathrm{PA}_{\mathrm{A}}$ in methanol. The $\mathrm{PA}_{\mathrm{C}}$ layer is insoluble in methanol preventing its redissolution. The spin-coating procedure yields approximately $150 \mathrm{~nm}$ films of each layer. It is carried out in air resulting in about 15 minutes of air exposure of the $\mathrm{PA}_{\mathrm{C}}$ casting solution/film and approximately 5 minutes of air exposure for the $\mathrm{PA}_{\mathrm{A}}$ casting solution/forming bilayer film.

The use polyelectrolytes in a bipolar membrane geometry is necessary to the operational mechanism of Fig. 1 of the main text. For the system to retain counter-ions that can be released upon photoexcitation, the regions of bound cations and anions must be sufficiently separated to prevent salt from dissolving out of the SBM absent photoexcitation. For instance, if $\mathrm{PA}_{\mathrm{A}}$ and $\mathrm{PA}_{\mathrm{C}}$ were intimately blended together, their fixed counterions could directly compensate each other, and the $\mathrm{AgNO}_{3}$ would simply dissolve out of the film when contacted with $\mathrm{CH}_{3} \mathrm{CN}$. Retaining counter ions in the bipolar membrane requires that the energetic cost for separating the bound cation and anion must be greater than the solubility driving force for the transfer of counter ions to a contacting phase.

\subsection{Ion-exchanging SBM}

After spin coating, the bilayer modified glass slide was clamped to a No. 15 O-ring joint that had been added to the bottom of a standard three neck flask (see Figure S1). The flask was purged with nitrogen and covered to prevent unintentional light exposure. Such an environment was maintained throughout the experiments. A $0.3 \mathrm{M}$ solution of $\mathrm{AgNO}_{3}$ in $\mathrm{CH}_{3} \mathrm{CN}$ was added to the flask, and the bilayer film was left to soak in this solution for 60 minutes. All $\mathrm{CH}_{3} \mathrm{CN}$ used was HPLC grade and thoroughly $\mathrm{N}_{2}$ sparged. Following removal of the $\mathrm{AgNO}_{3}$ solution, the film was rinsed three times with $\mathrm{CH}_{3} \mathrm{CN}$. During each rinse the $\mathrm{CH}_{3} \mathrm{CN}$ was left in contact with the bilayer for at least 30

*lonergan@uoregon.edu 


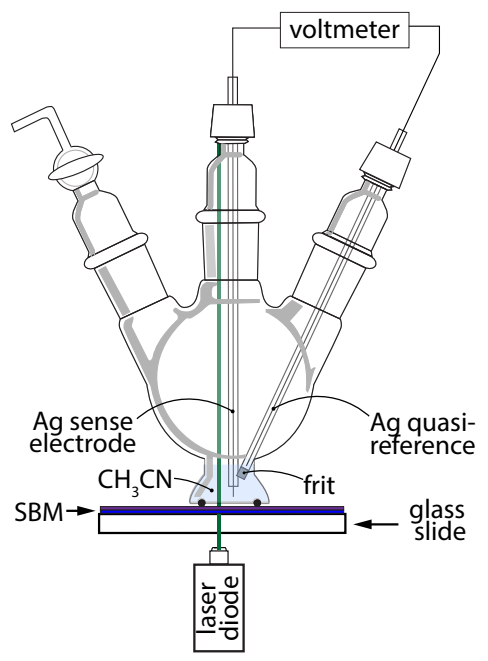

Figure S1: Schematic of cell used for completing ion-exchange and measuring the electrode potential change in response to illumination. The same structure absent the Ag sense and Ag quasi-reference electrodes was also used for illumination studies that were then analyzed by ICP-MS.

minutes before removing. It is noted that the tetramethylammonium salt of $\mathrm{PA}_{\mathrm{A}}$ dissolves slowly in acetonitrile, but the ion exchange with $\mathrm{Ag}^{+}$rendered it insoluble, and no dissolution was observed.

\subsection{Electrode potential measurements}

One port of the three-neck flask to which the SBM had been attached was fitted with the sensing silver wire apparatus consisting of a bare $2 \mathrm{~mm}$ portion of a silver wire extending from a glass tube in which the remainder of the wire had been sealed off with epoxy (see Fig. S1). A second port was fitted with a the reference silver wire assembly consisting of a glass tube to which a porous glass frit had been attached using teflon heat shrink tubing, and into which a silver was had been inserted. The third port remained a port for maintaining a nitrogen environment. Before each use, both silver wires were prepared as quasi-reference electrodes by first soaking in $10 \%(\mathrm{w} / \mathrm{v}) \mathrm{HCl}$ for fifteen minutes followed by bleach for 15 minutes, and then rinsing first with water and then $\mathrm{CH}_{3} \mathrm{CN}$.

To measure the response of the bilayer, $0.3 \mathrm{~mL}$ of $\mathrm{CH}_{3} \mathrm{CN}$ was added to the main compartment of the three-neck flask so that it was in contact with both the bilayer film, the frit of the reference silver wire assembly, and the exposed silver wire tip of the sensing silver wire assembly. The reference compartment was also filled with $\mathrm{CH}_{3} \mathrm{CN}$. The potential of the sensing silver wire versus the reference silver wire was monitored using a Philips PM2534 digital multimeter. The potential was first allowed to stabilize to a drift of less than $0.5 \mathrm{mV} / \mathrm{min}$, which typically took between 20 minutes and two hours. It is not clear why there was such a large variation in the time it took for the system to stabilize. Once stabilized, the potential was recorded for 2 minutes. Then while continuing to record, a portion of the bilayer was illuminated through the glass slide substrate with an approximately $1.5 \mathrm{~mm}$ diameter spot of $1 \mathrm{~mW}, 532 \mathrm{~nm}$ light from a Lasermate GML-523 laser diode for two minutes. The $532 \mathrm{~nm}$ light is on the high energy side of the absorption maximum of $\mathrm{PA}_{\mathrm{A}}$ and $\mathrm{PA}_{\mathrm{C}}$, which is at $620 \mathrm{~nm} .{ }^{1}$ The potential was then recorded for an additional two 

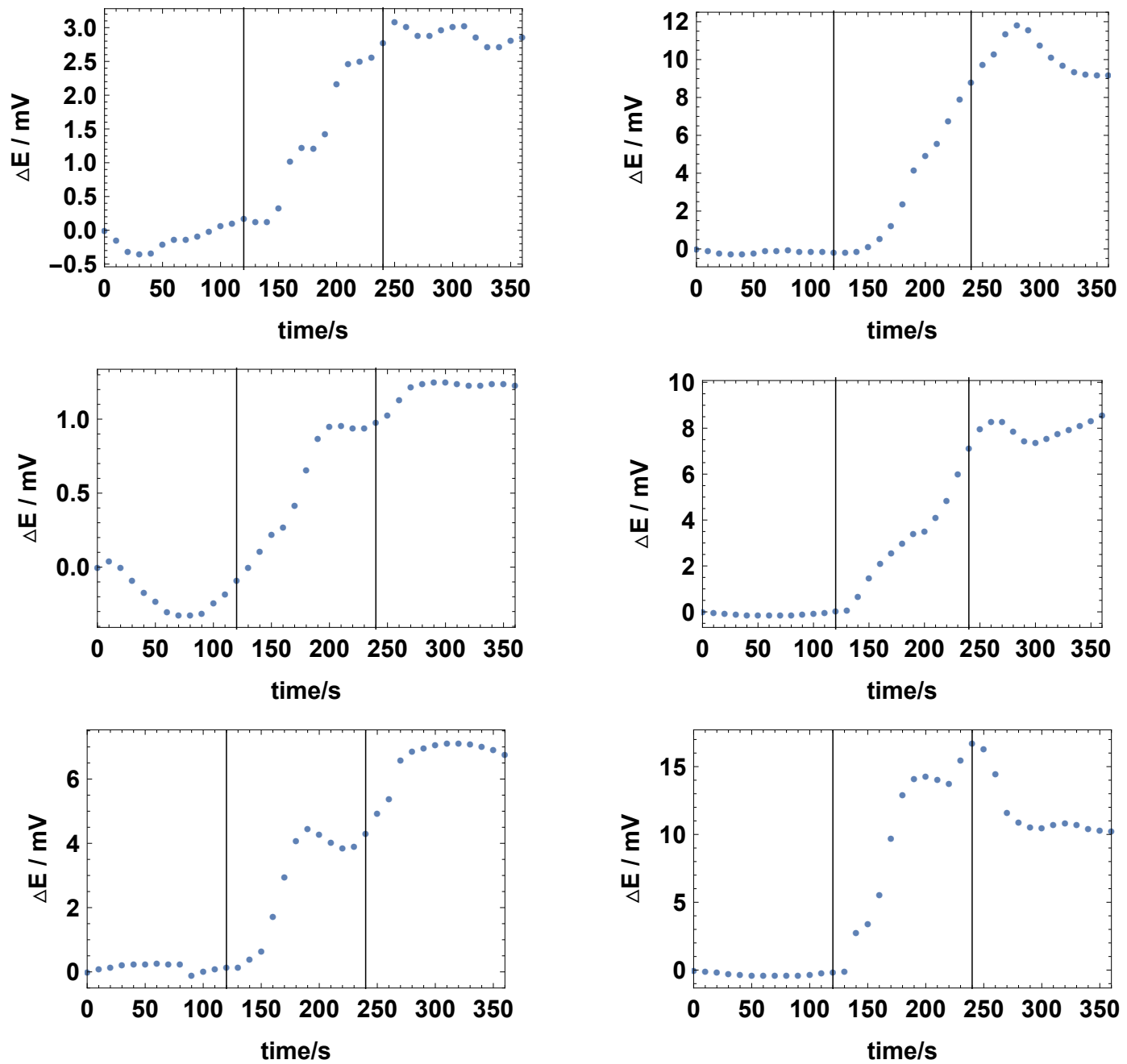

Figure S2: Change in electrode potential versus time for the six measurements on one $\mathrm{PA}_{\mathrm{A}} / \mathrm{PA}_{\mathrm{C}}$ SBM. Illumination was between time points $\mathrm{t}=120 \mathrm{~s}$ and $240 \mathrm{~s}$ as marked by the vertical lines. Note the different vertical scaling on the different plots.

minutes following illumination. The silver wire assemblies were kept out of the path of the laser illumination, and the tip of the sensing wire was up to $5 \mathrm{~mm}$ away from the illumination spot. For measurements on the same bilayer, the $\mathrm{CH}_{3} \mathrm{CN}$ was replaced, the potential allowed to stabilize and the illumination spot moved to an unexposed area before each new illumination cycle. Fig. S2 and S3 show the twelve individual measurements conducted on two separate bilayer films. Illumination of a clean glass slide with no polymer layer in the experimental geometry described resulted in no measurable change in electrode potential. 

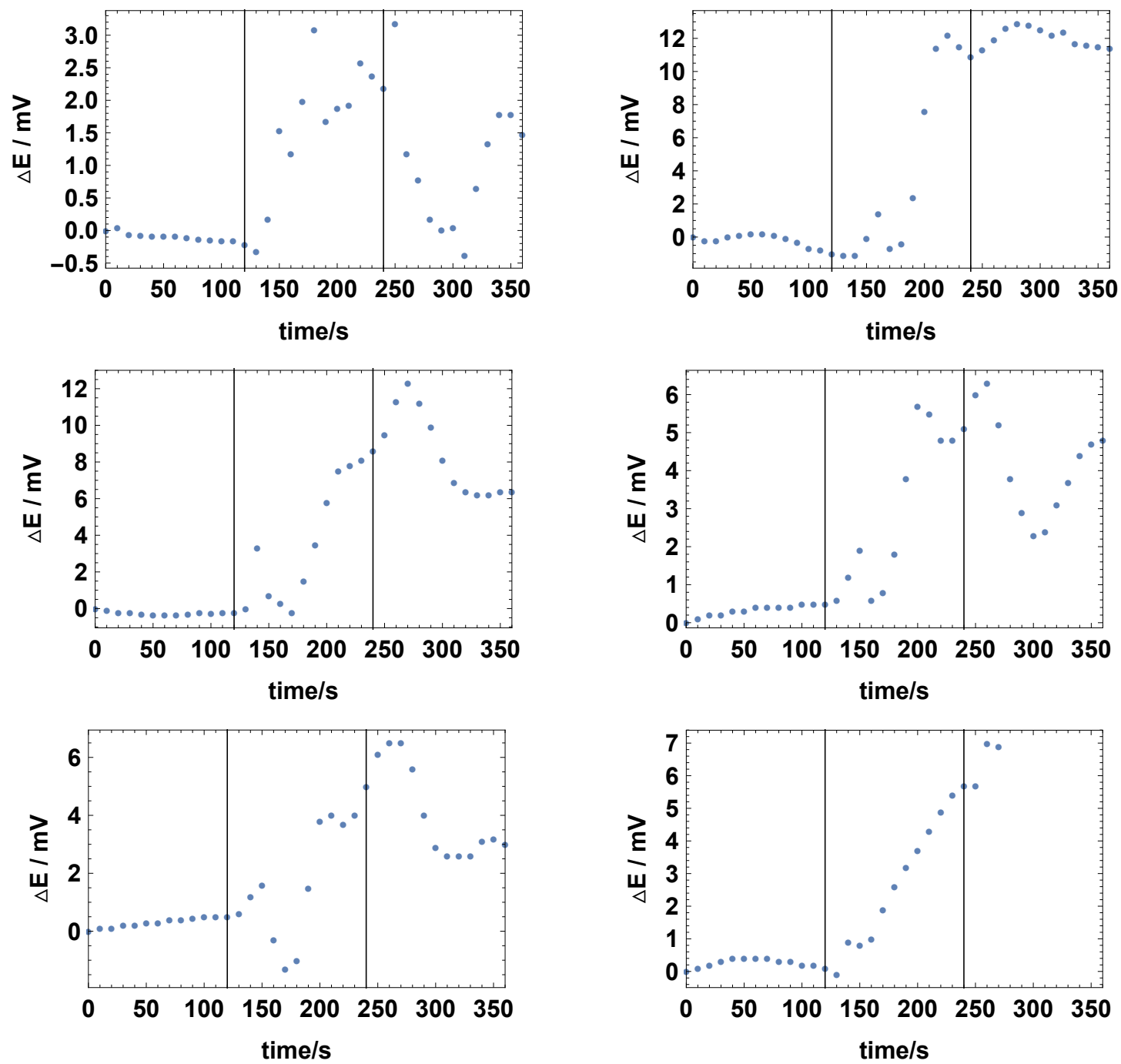

Figure S3: Change in electrode potential versus time for the six measurements on a different $\mathrm{PA}_{\mathrm{A}} / \mathrm{PA}_{\mathrm{C}} \mathrm{SBM}$ than in Fig. S2. Illumination was between time points $\mathrm{t}=120 \mathrm{~s}$ and $240 \mathrm{~s}$ as marked by the vertical lines. During the bottom right measurement, the electrode assembly was accidentally disturbed at $\mathrm{t}=260 \mathrm{~s}$, which is why only data up to that time point are shown. Note the different vertical scaling on the different plots. 


\subsection{ICP-MS measurements}

The same cell in Fig. S1 without the silver sense and quasi-reference electrodes was used for measuring $\mathrm{Ag}^{+}$release by inductively coupled plasma-mass spectrometry (ICP-MS). The PAA $/ \mathrm{PA}_{\mathrm{C}}$ $\mathrm{SBM}$ was prepared and ion exchanged as described above. An aliquot of $0.3 \mathrm{~mL}$ of $\mathrm{CH}_{3} \mathrm{CN}$ was then added to the flask and allowed to remain for two minutes in the dark before it was transferred into a scintillation vial. A second aliquot of the same volume was then added, and a $1.5 \mathrm{~mm}$ diameter spot illuminated with a $1 \mathrm{~mW}$ of green light $(532 \mathrm{~nm})$ in the same manner as was done for the electrode potential measurements. The illumination was carried out for two minutes after which the $\mathrm{CH}_{3} \mathrm{CN}$ was transferred to a separate scintillation vial. A third aliquot was then added and the two minute illumination repeated and the $\mathrm{CH}_{3} \mathrm{CN}$ transferred to a third scintillation vial. The $\mathrm{CH}_{3} \mathrm{CN}$ was evaporated from each vial with a stream of $\mathrm{N}_{2}$, and any residue was dissolved in $2 \%$ $(\mathrm{w} / \mathrm{w}) \mathrm{HNO}_{3}(\mathrm{aq})$. The $2 \% \mathrm{HNO}_{3}(\mathrm{aq})$ was prepared by dilution of Certified ACS plus $70 \%$ nitric acid from Fisher Scientific with $18 \mathrm{M} \Omega \mathrm{cm}$ water. Each vial was analyzed using a ThermoFisher iCAP RQ ICP-MS. The Ag content was quantified by comparison to standards prepared from $\mathrm{AgNO}_{3}$ dissolved in $2 \% \mathrm{HNO}_{3}(\mathrm{aq})$. The samples were in the range 0-100 ppb with standards of 10, 20, 50 and $100 \mathrm{ppb}$ used for the determination. The experiment was repeated on three more $\mathrm{PA}_{\mathrm{A}} / \mathrm{PA}_{\mathrm{C}} \mathrm{SBM}$ membrane samples to provide a total of 8 illumination cycles and 4 dark cycles. The same experiment was also conducted on two single layer films of each of $\mathrm{PA}_{\mathrm{A}}$ and $\mathrm{PA}_{\mathrm{C}}$ for four illumination cycles and two blank cycles on each. For the single layer samples, some negative concentrations were recorded reflecting the detection limit of the experiment given background fluctuations, potential matrix effects, and the quality of the standards.

Figure S4 shows the average results of ICP-MS analysis and Fig. S5 the individual measurements. The solvent layer from the illuminated $\mathrm{PA}_{\mathrm{A}} / \mathrm{PA}_{\mathrm{C}} \mathrm{SBM}$ membranes shows significantly more silver than absent illumination or that observed for illuminated single $\mathrm{PA}_{\mathrm{A}}$ and $\mathrm{PA}_{\mathrm{C}}$ layers. Negative masses were determined for aliquots from some of the single layer films and are a consequence of the detection limit of the experiment, which involved referencing to blank (nominally no $\mathrm{Ag}$ ) and standard samples. The expected mass of $\mathrm{Ag}$ for the $\mathrm{PA}_{\mathrm{A}} / \mathrm{PA}_{\mathrm{C}} \mathrm{SBMs}$ can be approximated by estimating the density of the silver salt of $\mathrm{PA}_{\mathrm{A}}$ as $2 \mathrm{~g} / \mathrm{cm}^{3}$. For comparison, the densities of silver nitrate and silver acetate are $4.35 \mathrm{~g} / \mathrm{cm}^{3}$ and $3.26 \mathrm{~g} / \mathrm{cm}^{3}$, respectively. Based on the density estimate, the mass of silver ion in a $1.5 \mathrm{~mm}$ diameter section of a $150 \mathrm{~nm}$ thick film of the silver salt of $\mathrm{PA}_{\mathrm{A}}$ is $0.2 \mu \mathrm{g}$. It is also possible that the potential silver ion available for release is much greater because this estimate does not account for the radial diffusion of ions and electronic carriers to and from the illumination area, which is only a spot on a larger film. The fraction of available $\mathrm{Ag}^{+}$that could potentially be pumped out of the system will be discussed in the context of numerical simulations below.

\section{Numerical Simulations}

\subsection{Computational details}

Numerical simulations were performed using the transport of diluted species and electrostatics modules of COMSOL Multiphysics, Version 4.4. Many of the symbols for the parameters used and their values are shown in Table S1. A one-dimensional model with position variable $x$ was used as shown in Fig. S6. The SBM ran from $x=0$ to $x=2 l$ with each layer being of thickness $l$, and the solvent from $x=2 l$ to $x=2 l+l^{(s)}$. The ratio of the thickness of the solvent layer to the 


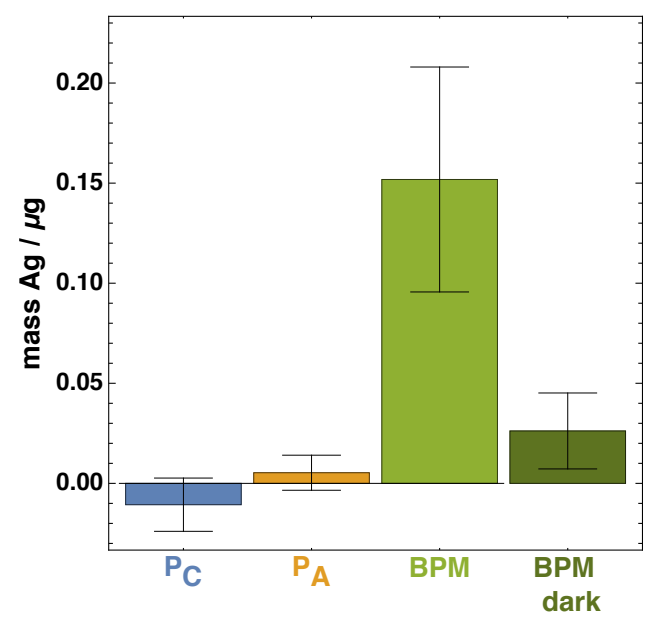

Figure S4: Average mass of silver in the solvent layer determined by ICP-MS following a 2 minute illumination of a $1.5 \mathrm{~mm}$ spot of single layer $\mathrm{PA}_{\mathrm{C}}$ films (blue), single layer $\mathrm{PA}_{\mathrm{A}}$ films (orange), and $\mathrm{PA}_{\mathrm{A}} / \mathrm{PA}_{\mathrm{C}} \mathrm{SBMs}$ (light green). The $\mathrm{PA}_{\mathrm{A}}$ and $\mathrm{PA}_{\mathrm{C}}$ results are for two illumination cycles each on two separate films of each kind, and the $\mathrm{PA}_{\mathrm{A}} / \mathrm{PA}_{\mathrm{C}}$ data are for two illumination cycles each on four separate SBMs. For comparison, results for solvent layers contacted to the four $\mathrm{PA}_{\mathrm{A}} / \mathrm{PA}_{\mathrm{C}} \mathrm{SBMs}$ in the dark are also shown (dark green). In each case, the bar height is the average of and the error bars the standard deviation of the trials.

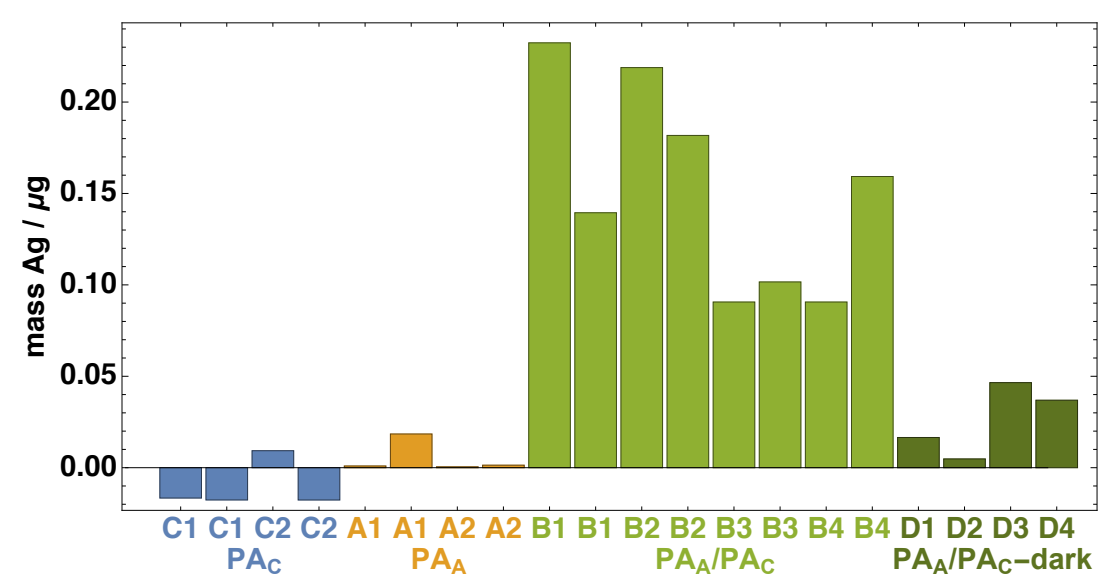

Figure S5: Individual measurements of the mass of Ag determined by ICP-MS analysis in solvent layers contacted to $\mathrm{PA}_{\mathrm{C}}$ (blue, "C" samples) and $\mathrm{PA}_{\mathrm{A}}$ (orange, "A" samples) single layer samples and $\mathrm{PA}_{\mathrm{A}} / \mathrm{PA}_{\mathrm{C}}$ bilayer samples (light green, "B" samples) after two minutes of illumination. For comparison, the $\mathrm{Ag}$ content of solvent layers after contacting $\mathrm{PA}_{\mathrm{A}} / \mathrm{PA}_{\mathrm{C}}$ bilayers in the dark (dark green, "D" samples) is also shown. There are two measurements for each sample label corresponding to the analysis of solvent layers from two separate illumination cycles on different spots of the film. 


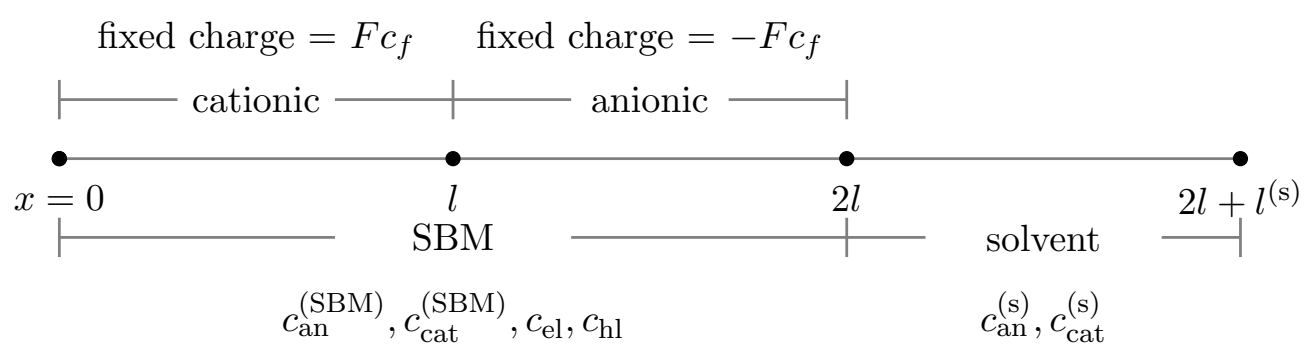

Figure S6: Schematic of 1D COMSOL model. The SBM runs from $x=0$ to $x=2 l$ and treats particle concentrations for anions, cations, electrons and holes as indicated at the bottom of the figure. The solvent runs from $x=2 l$ to $x=2 l+l^{(s)}$ and treats particle concentrations for anions and cations. The cationic portion of the active region runs from $x=0$ to $x=l$, and in this region, a positive space charge of $F c_{o}$ contributes to the excess charge density, as indicated at the top of the figure. The anionic portion of the active region runs from $x=l$ to $x=2 l$, and in this region, a negative space charge of $-F c_{o}$ contributes to the excess charge density.

SBM is $\eta=l^{(\mathrm{s})} / l$. Both the solvent and SBM contained the physics for anions and cations, but electrons and holes were confined to the SBM. The anion and cation populations in the SBM and solvent were separate, related by boundary reactions in their respective continuity equations (see below). Hence, the concentration of the anions and cations could in general be discontinuous at the SBM/solvent boundary. Herein, a general subscript $i$ is used as a particle label: an = anion, cat $=$ cation, el $=$ electron, $\mathrm{hl}=$ hole, and for the anions and cations a superscript $(j)$ is used to indicate the SBM $(j=(S B M))$ or solvent $(j=(s))$ layer ion concentrations where a distinction is necessary. The full set of parameters, the symbols used for them, and the values used in the simulations are listed in Table S1. The gas constant is $R$, and the Faraday constant is $F$.

The default finite element scheme in COMSOL was used to discretize the drift/diffusion equations for the partial particle currents $J_{i}$ in the bulk of each region:

$$
J_{i}=-z_{i} F D_{i} \frac{d c_{i}}{d x}-\sigma_{i} \frac{d \phi}{d x}
$$

Here, $\phi$ is the electrostatic potential; $c_{i}, z_{i}$, and $D_{i}$ are the concentration, charge number, and diffusion coefficient of particle $i ; \sigma_{i}=\left|z_{i}\right| F c_{i} \mu_{i}$ is the conductivity of particle $i$. The Einstein relation, $D=\mu R T / F$, was used to related $D$ and $\mu$. The drift/diffusion equations were solved together with Poisson's equation and the continuity equations. The former is given by:

$$
\epsilon \frac{d^{2} \phi}{d x^{2}}=\rho
$$

where $\epsilon$ is the dielectric constant. The excess charge density $\rho$ is given by:

$$
\rho= \begin{cases}F\left(c_{c a t}^{(\mathrm{SBM})}+c_{h}-\left(c_{a n}^{(\mathrm{SBM})}+c_{e}\right)+c_{f}\right), & 0 \leq x<l \\ F\left(c_{\text {cat }}^{(\mathrm{SBM})}+c_{h}-\left(c_{a n}^{(\mathrm{SBM})}+c_{e}\right)-c_{f}\right), & l \leq x \leq 2 l \\ F\left(c_{c a t}^{(\mathrm{s})}-c_{a n}^{(\mathrm{s})}\right), & 2 l \leq x \leq 2 l+l^{(\mathrm{s})}\end{cases}
$$

where $c_{f}$ is the concentration of fixed charge in the SBM. This continuum of fixed charge is what is used to model the presence of ionic functional groups in the ionically functionalized conjugated 
Table S1: Parameters for 1D simulations

\begin{tabular}{l}
\hline Symbol \\
\hline$T$ \\
SBM \\
$G$ \\
$\beta$ \\
$\epsilon^{(\mathrm{SBM})}$ \\
$D_{a n}$ \\
$D_{c a t}$ \\
$D_{e l}$ \\
$D_{h l}$ \\
$c_{f}$ \\
$l$
\end{tabular}

Description

temperature

generation rate (varied)

radiative recomb. coeff.

dielectric constant (varied)

anion diff. coefficient (varied)

cation diff. coefficient (varied)

electron diffusion coefficient

hole diffusion coefficient

fixed charge conc. (varied)

thickness of each layer of SBM
Value

293.15

\section{Solvent}

$\epsilon^{(\mathrm{s})}$

dielectric constant

$25 \epsilon_{0}$

$D_{a n}$

$D_{\text {cat }}$

$l^{(\mathrm{s})}$

anion diffusion coefficient

$1 \times 10^{-9} \mathrm{~m}^{2} \mathrm{~s}^{-1}$

cation diffusion coefficient

$1 \times 10^{-9} \mathrm{~m}^{2} \mathrm{~s}^{-1}$

thickness of solvent (varied)

$1,10,100 \mathrm{~nm}$

up to $223 \mathrm{M} \mathrm{s}^{-1}=$

$5 \times 10^{10} \mathrm{M}^{-1} \mathrm{~s}^{-1}$

$5 \epsilon_{0}$ and $25 \epsilon_{0}$

$1 \times 10^{-9}$ and $1 \times 10^{-10} \mathrm{~m}^{2} \mathrm{~s}^{-1}$

$1 \times 10^{-9}$ and $1 \times 10^{-10} \mathrm{~m}^{2} \mathrm{~s}^{-1}$

$1 \times 10^{-9} \mathrm{~m}^{2} \mathrm{~s}^{-1}$

$1 \times 10^{-9} \mathrm{~m}^{2} \mathrm{~s}^{-1}$

$0.001-0.1 \mathrm{M}$

$100 \mathrm{~nm}$

\section{SBM/solvent Boundary}

$K=k_{f} / k_{b}$

1 to 10000 
polymers, and hence it adds in the excess charge density expression in the region corresponding to the cationic polymer $(0 \leq x<l)$ and subtracts in the region corresponding to the anionic polymer $(l \leq x<2 l)$. The continuity equations for the electrons and holes, which are confined to the SBM, are:

$$
\frac{d c_{i}}{d t}=-\frac{1}{z_{i} F} \frac{d J_{i}}{d x}+G-\beta c_{e l} c_{h l}
$$

where $G$ is the generation rate created by photoexcitation and $\beta$ is the direct recombination rate constant. The direct recombination rate used is that corresponding to the radiative recombination limit of a traditional semiconductor with a band gap of $1.5 \mathrm{eV}$. The continuity equations for the anions and cations in the SBM are:

$$
\frac{d c_{i}^{(\mathrm{SBM})}}{d t}=-\frac{1}{z_{i} F} \frac{d J_{i}}{d x}-k_{f} c_{i}^{(\mathrm{SBM})}(x=2 l)+k_{b} c_{i}^{(\mathrm{s})}(x=2 l)
$$

where $k_{f}$ is the rate constant for transfer of ions from the SBM to the solvent (taken as the same for anions and cations) and $k_{b}$ is that for the reverse transfer. The continuity equations for the anions and cations in the solvent are:

$$
\frac{d c_{i}^{(\mathrm{s})}}{d t}=-\frac{1}{z_{i} F} \frac{d J_{i}}{d x}+k_{f} c_{i}^{(\mathrm{SBM})}(x=2 l)-k_{b} c_{i}^{(\mathrm{s})}(x=2 l)
$$

The drift/diffusion, continuity, and Poisson's equations were solved in COMSOL subject to the following constraints. The total number of anions and cations throughout the structure was conserved:

$$
\begin{aligned}
& \int_{0}^{2 l+l^{(\mathrm{s})}} c_{a n} d x-c_{f} l=0 \\
& \int_{0}^{2 l+l^{(\mathrm{s})}} c_{c a t} d x-c_{f} l=0
\end{aligned}
$$

The number of electrons was kept equal to the number of holes:

$$
\int_{0}^{2 l}\left(c_{e l}-c_{h l}\right) d x=0
$$




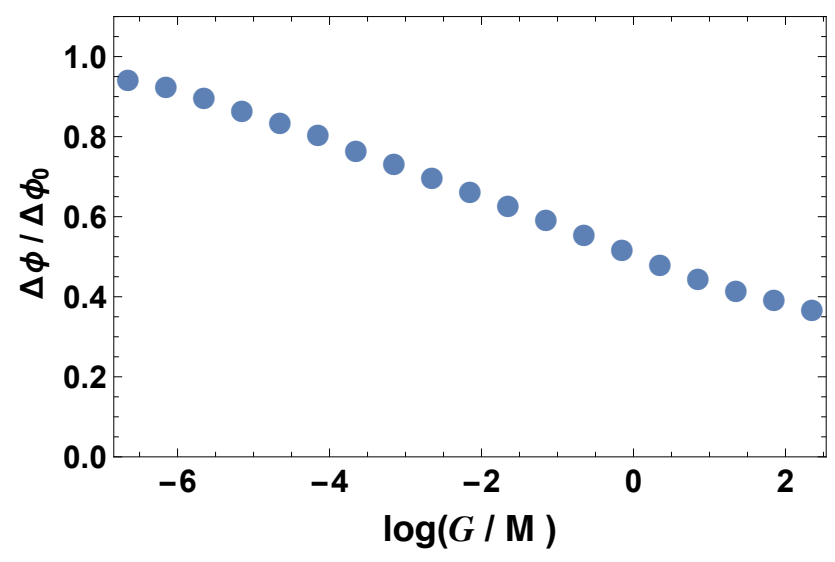

Figure S7: Electrostatic potential difference $\Delta \phi$ between the side of the cationic layer away from the solvent $(x=0)$ and the boundary of the solvent away from the SBM $(x=0)$ as a function of $G$ for the SBM with the same parameters as the blue circles in Fig. 6 of the main text. The $\Delta \phi$ is normalized by the value with no electronic carrier generation $\Delta \phi_{0}$

\subsection{Additional simulation data}

\subsubsection{Electrostatic potential}

Figure S7 shows the light intensity dependence of the electrostatic potential difference between the body of the cationic layer (more precisely at $x=0$ ) and the boundary of the solution away from the SBM $\left(x=2 l+l^{(\mathrm{s})}\right)$ normalized by this difference for $G=0$. As can be seen, the potential difference decreases with increasing $G$. This is because an increasing number of fixed counterions in the SBM become compensated by electronic charge carriers, which do not participate in the Donnan equilibrium because they cannot partition into the solvent.

\subsubsection{The reduction scheme and variation with $K, c_{f}$, and $l^{(s)}$}

The data of Fig. 4a in the main manuscript can be reduced as shown in Fig. S8 by first considering $c_{b}^{(\mathrm{s})}$ in the $G \rightarrow 0$ and $G \rightarrow \infty$ limits. The former is assigned the symbol $c_{0}^{(\mathrm{s})}$ and is determined by the numerical simulations. The large $G$ limit of $c_{b}^{(\mathrm{s})}$ is proposed to be that determined by $K$ absent any space charge effects. The concentrations in the solvent and $\operatorname{SBM}\left(c_{K}^{(\mathrm{s})}\right.$ and $c_{K}^{(\mathrm{SBM})}$, respectively) in this situation are determined by $K=c_{K}^{(s)} / c_{K}^{(S B M)}$ and the conservation of mass requirement $l^{(\mathrm{s})} c_{K}^{(\mathrm{s})}+l c_{K}^{(\mathrm{SBM})}=l c_{f}$ yielding:

$$
\lim _{G \rightarrow \infty} c_{b}^{(\mathrm{s})}=c_{K}^{(\mathrm{s})}=K c_{K}^{(\mathrm{SBM})}=\frac{c_{f} K}{1+\eta K}
$$

where $\eta=l^{(\mathrm{s})} / l$. The $c_{0}^{(\mathrm{s})}$ and $c_{K}^{(\mathrm{s})}$ are expected to be the lower and upper bounds, respectively, over which $c_{b}^{(s)}$ can vary. If this is indeed the case, the quantity $\left(c_{b}^{(s)}-c_{0}^{(s)}\right) /\left(c_{K}^{(s)}-c_{0}^{(s)}\right)$ is expected to run from 0 to 1 . The generation axis in Fig. $4 \mathrm{~b}$ of the main text is reduced as $\sqrt{G / \beta} / c_{K}^{(\mathrm{SBM})}$.

Figures S9-S11 show additional simulation data exploring the reduced variable scheme proposed in the paper. Figure S9 shows the result of systematically varying $K$. In Part (a) it can be seen 


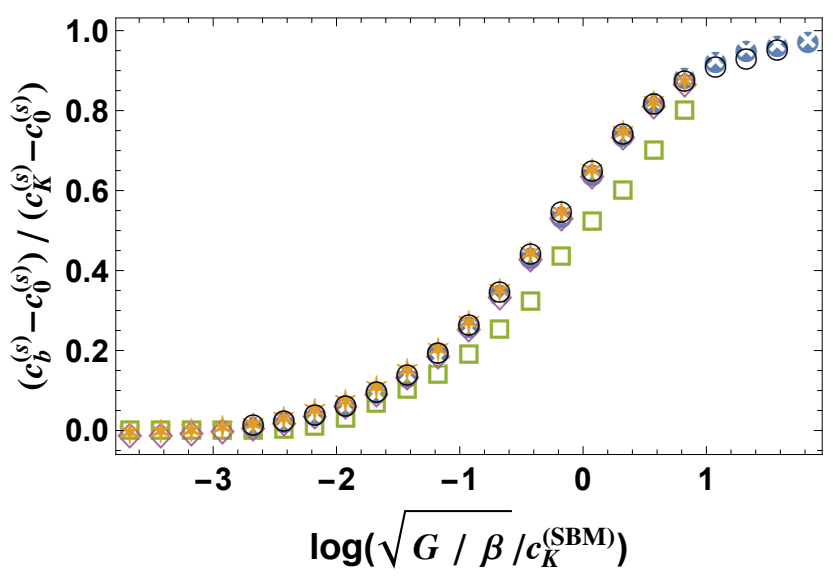

Figure S8: Simulation data from Figure 4a of the main paper reduced as described in the text.

that for the largest value of $\mathrm{K}=10000$, the highest illumination level is able to drive nearly all of the ions out of the SBM with $c_{b}^{(\mathrm{s})}$ approaching $c_{f}=0.01 M$, which is the expected maximum solvent concentration because $\eta=1$. The ions are simply shifted from one layer of the SBM to an equal volume of solvent. As $K$ is decreased, the propensity for ions to be driven into the solvent decreases, and it takes increasing illumination levels to drive an increase in $c_{b}^{(\mathrm{s})}$ (i.e. the curves shift to the right). Figure S9b shows the simulation data reduced as described in the main paper, which results in all of the curves nearly collapsing onto the same universal curve. Figure S10 shows the effect of varying $c_{f}$. Here, the data in Part (a) is normalized by $c_{f}$. The blue circles are the same data as the blue circles in the earlier Fig. S9. The larger the concentration of ions initially in the SBM (the larger the $c_{f}$ ), the larger the illumination level needed to drive $c_{b}^{(\mathrm{s})}$ to its saturation level. Of course, this saturation level is also correspondingly higher. Again, the proposed reduction scheme results in the curves collapsing onto a universal curve as shown in Fig. S10b, although the agreement is not as good at the higher concentration of $c_{f}=0.1 \mathrm{M}$. Finally, Fig. S11 shows the effect of changing the thickness of the solvent layer (i.e. changing $\eta$ ) with $l^{(s)}=10 \mathrm{~nm}$ (orange circles), $100 \mathrm{~nm}$ (blue circles), and $1000 \mathrm{~nm}$ (green diamonds). Again, the blue circles are the same data as in Figs. S9 and S10. The data of Fig. S11 are normalized by $c_{f}$ and $\eta$. The smaller the value of $l^{(s)}$, the lower the illumination level needed to drive the solvent concentration to its maximum value, which in this case is $c_{f} / \eta$ because the SBM and solvent layers are not necessarily the same volume. As the solvent layer is made thinner, it is possible to drive the solvent concentration to significantly higher levels than the initial concentration the SBM, but a larger $G$ is needed to reach the maximum level. For instance, in the orange squares, the $c_{b}^{\mathrm{s}}$ approaches $0.1 \mathrm{M}$ with large $G$, as compared to $c_{f}=0.01 \mathrm{M}$. Again, Fig. S11b shows the data of Part (a) successfully reduced.

\section{On comparing the simulations and experimental polyacetylene system}

The material parameters used for the simulation are not expected to match those of the $\mathrm{PA}_{\mathrm{A}} / \mathrm{PA}_{\mathrm{C}}$ SBM. As not all of the relevant parameters are known, the simulations were carried out to understand the essential physics of the action of the SBM. With that said, it is still relevant to 

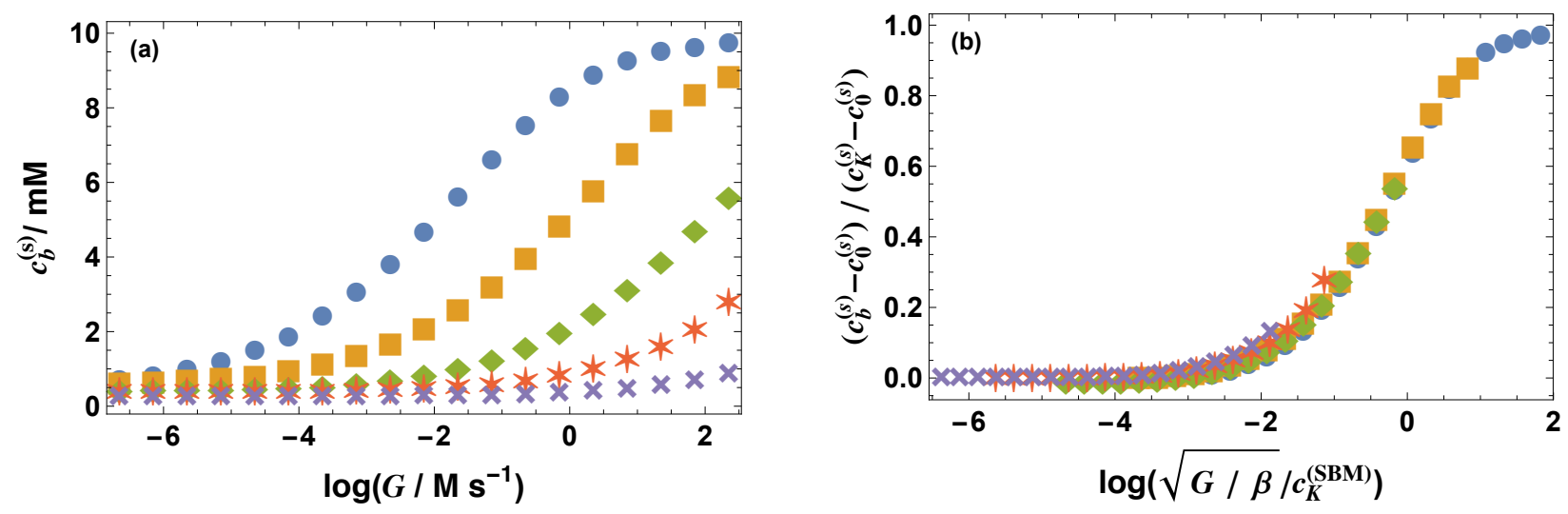

Figure S9: Part (a) shows simulation results of $c_{b}^{(\mathrm{s})}$ as a function of $G$ (illumination level) for a $\mathrm{SBM} /$ solvent system with $\epsilon^{(\mathrm{SBM})}=5, \epsilon^{(\mathrm{s})}=25, D_{\mathrm{el}}^{(\mathrm{SBM})}=D_{\text {ion }}^{(\mathrm{SBM})}=D_{\text {ion }}^{(\mathrm{s})}=1 \times 10^{-5} \mathrm{~cm}^{2} \mathrm{~s}^{-1}$, $c_{f}=0.01 \mathrm{M}, \beta=5 \times 10^{10} \mathrm{M}^{-1} \mathrm{~s}^{-1}$, and $l=l^{(\mathrm{s})}=100$. Four values of $K$ are shown: 1 (purple x's), 10 (red stars) , 100 (green diamonds), 1000 (orange squares), 10000 (blue circles). Part (b) shows the results of Part (a) in terms of reduced variables.
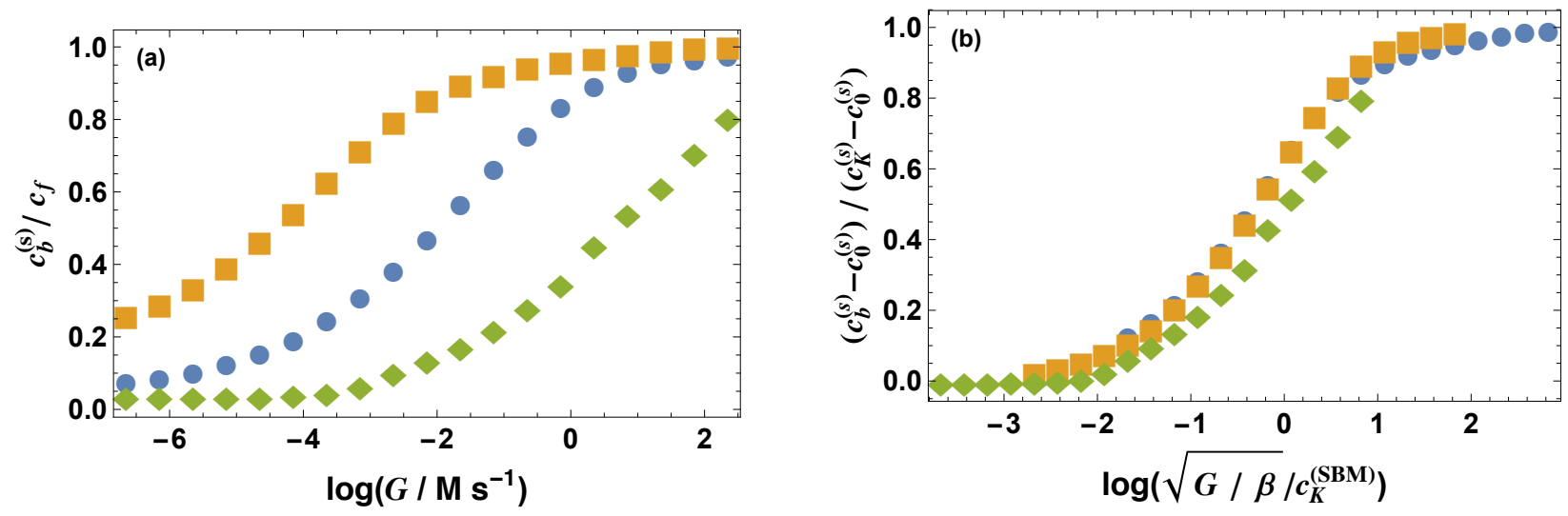

Figure S10: Part (a) shows simulation results of $c_{b}^{(\mathrm{s})}$ as a function of $G$ (illumination level) for a $\mathrm{SBM} /$ solvent system with $\epsilon^{(\mathrm{SBM})}=5, \epsilon^{(\mathrm{s})}=25, D_{\mathrm{el}}^{(\mathrm{SBM})}=D_{\text {ion }}^{(\mathrm{SBM})}=D_{\text {ion }}^{(\mathrm{s})}=1 \times 10^{-5} \mathrm{~cm}^{2} \mathrm{~s}^{-1}$, $\beta=5 \times 10^{10} \mathrm{M}^{-1} \mathrm{~s}^{-1}, l=l^{(\mathrm{s})}=100$, and $K=10000$. The concentrations are normalized by $c_{f}$, and three values of $c_{f}$ are shown: $0.001 \mathrm{M}$ (orange squares), $0.01 \mathrm{M}$ (blue circles), $0.1 \mathrm{M}$ (green diamonds). Part (b) shows the results of Part (a) in terms of reduced variables. 

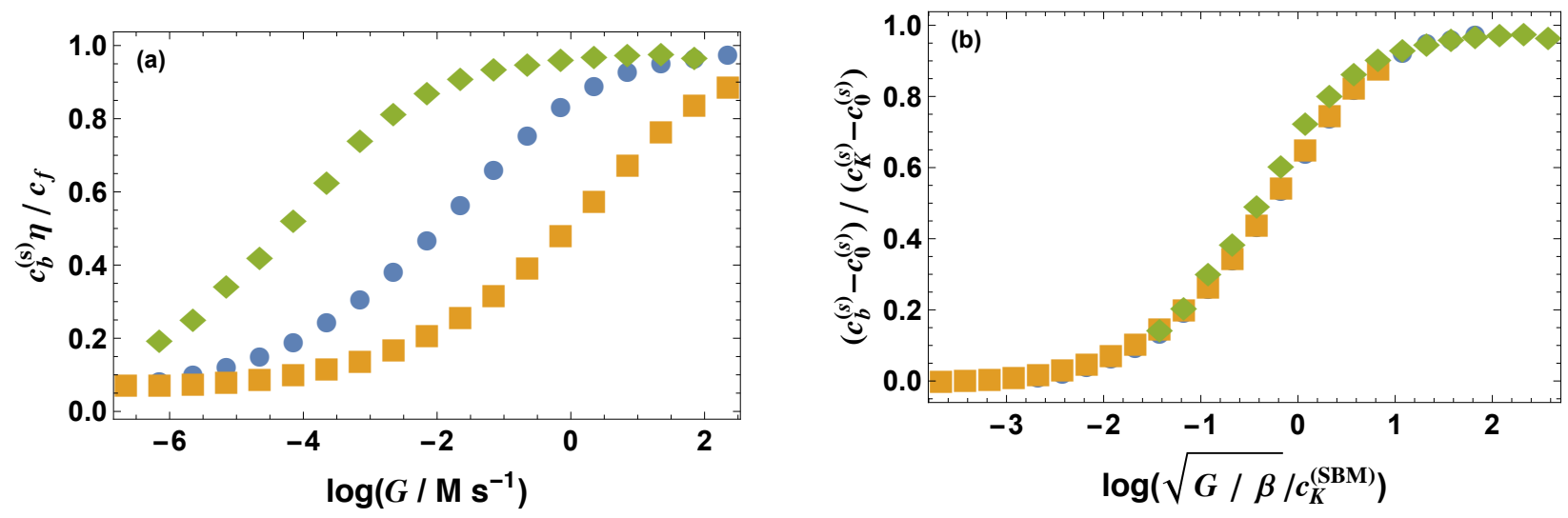

Figure S11: Part (a) shows simulation results of $c_{b}^{(\mathrm{s})}$ as a function of $G$ (illumination level) for a $\mathrm{SBM} /$ solvent system with $\epsilon^{(\mathrm{SBM})}=5, \epsilon^{(\mathrm{s})}=25, D_{\mathrm{el}}^{(\mathrm{SBM})}=D_{\text {ion }}^{(\mathrm{SBM})}=D_{\text {ion }}^{(\mathrm{s})}=1 \times 10^{-5} \mathrm{~cm}^{2} \mathrm{~s}^{-1}$, $c_{f}=0.01 \mathrm{M}, \beta=5 \times 10^{10} \mathrm{M}^{-1} \mathrm{~s}^{-1}, l=100 \mathrm{~nm}$, and $K=10000$. The results are normalized by $\eta / c_{f}$, and three values of $l^{(\mathrm{s})}$ are shown: $10 \mathrm{~nm}$ (orange squares, $\eta=0.1$ ), $100 \mathrm{~nm}$ (blue circles, $\eta=1$ ), $1000 \mathrm{~nm}$ (green diamonds, $\eta=10$ ). Part (b) shows the results of Part (a) in terms of reduced variables.

ask whether or not the simulations suggest that a significant fraction of the ions can be driven from the $\mathrm{PA}_{\mathrm{A}} / \mathrm{PA}_{\mathrm{C}} \mathrm{SBM}$, as observed experimentally, given likely discrepancies between the experimental and simulation parameters. First, the ionic conductivities ${ }^{2}$ of neat $\mathrm{PA}_{\mathrm{A}}$ and $\mathrm{PA}_{\mathrm{C}}$ $\left(10^{-11}-10^{-12} \mathrm{~S} / \mathrm{cm}\right)$ are much smaller than the ionic conductivities of liquid electrolytes where values of $D=1 \times 10^{-5} \mathrm{~cm}^{2} \mathrm{~s}^{-1}$ are more realistic. Second, the bound ion concentrations in the $\mathrm{PA}_{\mathrm{A}}$ and $\mathrm{PA}_{\mathrm{C}}$ layers are estimated to be on the order of $1 \mathrm{M}$ based on the formula weight and estimated density, whereas the simulations were performed in the 0.01 to $0.1 \mathrm{M}$ range because higher concentration simulations could not be consistently converged. Third, recombination in the $\mathrm{PA}_{\mathrm{A}}$ and $\mathrm{PA}_{\mathrm{C}}$ layers is likely to be significantly faster than in the radiative limit used in the simulations.

In regards to the first discrepancy, the steady-state simulation results are not sensitive to the value of $D_{\text {ion }}^{(\mathrm{SBM})}$ (see Fig. 6 of the main text), but a low $D_{\text {ion }}^{(\mathrm{SBM})}$ could impact the ability of ions to escape the film on the time scale of the experiment. This concern, however, is mitigated by the thinness of the films and the fact that they swell with $\mathrm{CH}_{3} \mathrm{CN}$, which greatly increases their conductivity. ${ }^{2}$ A diffusion length of $150 \mathrm{~nm}$ over $10 \mathrm{~s}$ only requires a diffusion coefficient of $2 \times 10^{-11} \mathrm{~cm}^{2} \mathrm{~s}^{-1}$. Further, cyclic voltammetry with micron thick $\mathrm{PA}_{\mathrm{C}}$ and $\mathrm{PA}_{\mathrm{C}}$ films swollen with $\mathrm{CH}_{3} \mathrm{CN}$ can be readily performed on the timescale of seconds, which involves the addition and removal of ions from the films. ${ }^{3}$ It is also possible that Donnan exclusion might hinder anion transport from the $\mathrm{PA}_{\mathrm{C}}$ bottom layer through the $\mathrm{PA}_{\mathrm{A}}$ top layer and into the solvent. The fact that the electronic charge carriers can render the individual layers charge neutral absent the mobile counterions might be expected to reduce any Donnan exclusion. In addition, cyclic voltammetry experiments on $\mathrm{PA}_{\mathrm{A}} / \mathrm{PA}_{\mathrm{C}}$ bilayers similarly prepared to those herein show that the anions from the bottom $\mathrm{PA}_{\mathrm{C}}$ layer can be driven from the film in support of electrochemical reduction. ${ }^{4}$

The variable reduction scheme of Fig. 6 in the main text helps in understanding the impact of the second two likely discrepancies mentioned above. Here, we make comparison to the system of the green squares in Fig. 6 of the main text where $c_{f}=0.1 \mathrm{M}$. The illumination intensity used in the experiments is approximately $50 \mathrm{~mW} \mathrm{~cm}^{-2}$, which corresponds to $\log (G / M) \approx 1$. This is just 
sufficient to drive the ions out of the film in the $c_{f}=0.1 \mathrm{M}$ simulation system. The still larger value of $c_{f}$ in the $\mathrm{PA}_{\mathrm{A}}$ and $\mathrm{PA}_{\mathrm{C}}$ films and the likely larger $\beta$, however, raises the question of whether or not all of the ions can be driven out of the film. The reduction scheme of Fig. 6 demonstrates that the light intensity needed to drive one half of the ions out of the film is approximately $\left(c_{K}^{(\mathrm{SBM})}\right)^{2} \beta$. According to eq 1 of the main text, the ten-fold larger $c_{f}$ in the experimental system relative to the simulation system contributes a factor of 10 increase to $c_{K}^{(\mathrm{s})}$ and $c_{K}^{(\mathrm{SBM})}$ and hence to apparently a 100 fold increase in the light intensity needed to drive a significant fraction of the ions out of the film. This is not the whole story, however. Another key difference between the simulations and experiments is the thickness of the solvent layer. The solvent layer is much thicker in the experiments with $\eta=2.6 \times 10^{4}$ as compared to $\eta=1$ used for most of the simulations. Even given the 10 fold increase in $c_{f}$, this results in $c_{K}^{(\mathrm{SBM})}$ being more than three orders of magnitude smaller in the experimental system. Hence, the recombination parameter $\beta$ could be more than six orders of magnitude larger than the radiative recombination limit and still have the experimental light intensity drive the majority of the ions from the film with the otherwise same material parameters as the simulations. The large $\eta$ could also compensate for a $K$ less than the 1000-10000 value used for most of the simulations. 


\section{References}

S1. Langsdorf, B. L.; Zhou, X.; Adler, D. H.; Lonergan, M. C. Synthesis and Characterization of Soluble, Ionically Functionalized Polyacetylenes. Macromolecules 1999, 32, 2796-2798.

S2. Lin, F.; Wang, Y.; Lonergan, M. Ion Transport in Polyacetylene Ionomers. J. Appl. Phys. 2008, $104,103517$.

S3. Lonergan, M. C.; Cheng, C. H.; Langsdorf, B. L.; Zhou, X. Electrochemical Characterization of Polyacetylene Ionomers and Polyelectrolyte-Mediated Electrochemistry toward Interfaces between Dissimilarly Doped Conjugated Polymers. J. Am. Chem. Soc. 2002, 124, 690-701.

S4. Robinson, S. G.; Lonergan, M. C. Polyacetylene p-n Junctions with Varying Dopant Density by Polyelectrolyte-Mediated Electrochemistry. J. Phys. Chem. C 2013, 117, 1600-1610. 